\title{
Article
}

\section{Single-Mode Lasing in Polymer Circular Gratings}

\author{
Saisai Chu ${ }^{1}$, Anwer Hayat ${ }^{2}$, Fengzhao Cao ${ }^{2}$ and Tianrui Zhai ${ }^{2, *(D)}$ \\ 1 State Key Laboratory for Mesoscopic Physics, Department of Physics, Peking University, \\ Beijing 100871, China; chusaisai@pku.edu.cn \\ 2 Institute of Information Photonics Technology, College of Applied Sciences, Beijing University of Technology, \\ Beijing 100124, China; anwerhayatnoor@gmail.com (A.H.); wincfz@emails.bjut.edu.cn (F.C.) \\ * Correspondence: trzhai@bjut.edu.cn; Tel.: +86-010-6739-2184
}

check for

updates

Citation: Chu, S.; Hayat, A.; Cao, F.; Zhai, T. Single-Mode Lasing in Polymer Circular Gratings. Materials 2021, 14, 2318. https://doi.org/ $10.3390 /$ ma14092318

Academic Editor: Aivaras Kareiva

Received: 14 March 2021

Accepted: 28 April 2021

Published: 29 April 2021

Publisher's Note: MDPI stays neutral with regard to jurisdictional claims in published maps and institutional affiliations.

Copyright: (c) 2021 by the authors. Licensee MDPI, Basel, Switzerland. This article is an open access article distributed under the terms and conditions of the Creative Commons Attribution (CC BY) license (https:// creativecommons.org/licenses/by/ $4.0 /)$.

\begin{abstract}
In recent years, conjugated polymers have become the materials of choice to fabricate optoelectronic devices, owing to their properties of high absorbance, high quantum efficiency, and wide luminescence tuning ranges. The efficient feedback mechanism in the concentric ring resonator and its circularly symmetric periodic geometry combined with the broadband photoluminescence spectrum of the conjugated polymer can generate a highly coherent output beam. Here, the detailed design of the ultralow-threshold single-mode circular distributed feedback polymer laser is presented with combined fabrication processes such as electron beam lithography and the spin-coating technique. We observe from the extinction spectra of the circular gratings that the transverse electric mode shows no change with the increase of incident beam angle. The strong enhancement of the conjugated polymer photoluminescence spectra with the circular periodic resonator can reduce the lasing threshold about $19 \mu \mathrm{J} / \mathrm{cm}^{2}$. A very thin polymer film of about $110 \mathrm{~nm}$ is achieved with the spin-coating technique. The thickness of the gain medium can support only the zero-order transverse electric lasing mode. We expect that such a low threshold lasing device can find application in optoelectronic devices.
\end{abstract}

Keywords: circular gratings; single-mode lasing; polymer lasers

\section{Introduction}

In optoelectronic and also in laser technology, gain materials are regarded as the building block that can improve device performance, and some of them open a new route towards real-life applications. Similarly, common gain materials, such as inorganic semiconductors, organic dyes, organic semiconductors, quantum dots, and perovskites, are used in laser systems with small differences in thin film fabrication properties, electrical conductivity, and difficulties in the manufacturing process [1-9]. Among them, organic semiconductors are composed of different element of chromophores with conjugated fragments. In organic semiconductors, conjugated polymers have magnificent optical and electrical properties, broad photoluminescence (PL) spectral in the visible region, lost-cost processing, and easy fabrication methods on the flexible substrates [3,10-13]. Therefore, all these unique and intriguing features of the conjugated polymers provide a new route to explore optoelectronic technologies and micro-cavity lasers [14,15]. In recent years, conjugated polymers have been extensively applied as gain mediums in laser systems with different optical feedback nanostructures, such as whispering gallery mode (WGM)[16,17], microdroplet [18], and distributed feedback (DFB) cavities [19-23]. Modern research considers many conjugated polymers in lasing applications, such as poly [9, 9-dioctylfluorenyl2,7-diyl]-end capped with DMP (PFO), poly[(9,9-dioctylfluorenyl-2,7-diyl)-alt-co-(1,4benzo- $\left(2,1^{\prime}, 3\right)$-thiadiazole $\left.)\right](\mathrm{F} 8 \mathrm{BT})$, and poly $\left[2\right.$-methoxy-5-( $3^{\prime}, 7^{\prime}$-dimethyloctyloxy)-1,4phenylenevinylene] (MDMO-PPV) covering the whole visible spectra, which can generate coherent light either pump optically or electrically [24-26].

Recently, various strategies have been proposed to engineer the operating characteristic of the second-order and high-order DFB polymer lasers, especially to control the 
output-coupled lasing beam (surface-emitting) with low divergence angle along and normal to the periodic nanostructures [27-29]. The performance of the DFB polymer lasers can be further improved by reducing the lasing threshold by manipulating the photonic mode density. Similarly, three-dimensional (3D) photonic band gap structures can be regarded as ideal candidates for attaining a low threshold by confining the photonic mode but the fabrication process and modification in structure are complicated. This problem can be resolved by adopting the circular grating structure already known from the literature [30-34]; the feedback mechanism in the cavity is similar to the second-order DFB lasers [35]. The circular periodic structures provide a nearly 2D feedback mechanism via second-order Bragg reflection process in the grating plane and simultaneously emit vertical radiation through first-order Bragg diffraction [36]. In circular periodic structures, in-plane momentum is conserved for radial directions and wave-guided mode leads to constructive interference of radiation out of the plane via Bragg scatterings. The Bragg diffraction process for the second-order DFB circular grating structures (CGS) can be written as [37]:

$$
k_{e} \sin \beta= \pm k_{g} \pm n G= \pm \frac{2 \pi n_{e f f}}{\lambda_{e}} \pm \frac{2 \pi n}{\Lambda}
$$

In the above equation, $k_{e}$ and $k_{g}$ are the emitted and guided-mode radiation wave vectors, whereas $G$ and $\beta$ are the Bragg grating vector and output coupling angle (angle of emission) related to the guided mode wave vector. The comprehensive section of the Equation (1) includes $n_{e f f}$ (effective refractive index), $\lambda_{e}$ (emission wavelength), $\Lambda$ (circular grating period), and $n$ (integer for grating order), respectively. Therefore, surfaceemitting lasing from the circular grating structure is obtained via first-order diffraction $(\beta=0)$ and the above equation can be further modified as; $\lambda_{\mathrm{e}}=n_{\text {eff }} \Lambda$. Similarly, the $2 \mathrm{D}$ feedback mechanism in the circular grating structures combine with the broadband emission spectrum of the conjugated polymer would be an excellent candidate for attaining high-performance, low-threshold, single-mode lasers with high $\mathrm{Q}$ factor in the visible region. To our knowledge, second-order circular grating structures with ultra-thin film of the conjugated polymer showing low threshold, $\mathrm{TE}_{0}$ mode, and single-mode lasing have not been reported yet.

In this article, we focus on the morphology and lasing performance of optically pumped surface-emitting circular DFB polymer laser. The solution of PMMA in chlorobenzene was spin-coated on a glass (BK7) substrate, and after the baking process circular grating structures were written with electron beam lithography (EBL). The gain material conjugated polymer F8BT was spin-coated on the prepared circular grating structures. Finally, the circular DFB polymer laser was optically pumped to investigate the lasing properties such as lasing thresholds and emission wavelength.

\section{Fabrication Methods and Materials}

In this work, we applied electron beam lithography, a simple and effective fabrication technique for writing the geometry of circular periodic structures on the glass substrate, which would provide essential feedback mechanism for attaining the surface-emitting circular DFB polymer laser. Figure 1 illustrates the whole schematic diagram of the optically pumped circular grating DFB polymer lasing device used in our experiment. Initially, a solution of electron-beam resist polymethylmethacrylate (PMMA) with a concentration of $4.0 \%$ in chlorobenzene was spin-coated on a glass substrate $(10 \mathrm{~mm} \times 10 \mathrm{~mm} \times 1 \mathrm{~mm})$. Similarly, we have achieved a PMMA film having a thickness of $160 \mathrm{~nm}$ at a spin-coating speed of $4000 \mathrm{rpm}$ for $30 \mathrm{~s}$. The prepared film of the electron-beam resist (PMMA) was baked on a hot plate at $150{ }^{\circ} \mathrm{C}$ for $2 \mathrm{~min}$. The same spin-coating technique was utilized to cover the electron beam resist film with $20 \mathrm{~nm}$ an anti-charging (AR-PC 5090.02, Allresist $\mathrm{GmbH}$, Strausberg, Germany) layer. Hence, the electron beam lithography (Raith e_LiNE plus, Brisbane, Australia) with an accelerating voltage of $30 \mathrm{kV}$ and working distance of 10 $\mathrm{mm}$ was utilized to write 2D circular periodic structures on the developed sample. After that, the sample was rinsed with DI water for removing the anti-charge layer and then 
developed with MIBK: IPA (1:3) and stopped with IPA for $30 \mathrm{~s}$, respectively. The schematic of the circular periodic nanostructures after the whole process is illustrated in the Figure 1a. Here, we used a conjugated polymer F8BT as a gain material, whose gain peak can be realized around $570 \mathrm{~nm}$ wavelength [38]. The gain material F8BT was dissolved in the xylene at a concentration of $23.5 \mathrm{mg} / \mathrm{mL}$. The solution of the F8BT was spin-coated on circular grating periodic nanostructures at $1500 \mathrm{rpm}$ for $30 \mathrm{~s}$. At this spin-coating speed, we realized approximately $110 \mathrm{~nm}$ thickened film of the F8BT on the circular grating structures. The final form of the circular grating DFB lasing device is schematically depicted in the Figure $1 \mathrm{~b}$. The actual composite photograph is shown in Figure 1c.
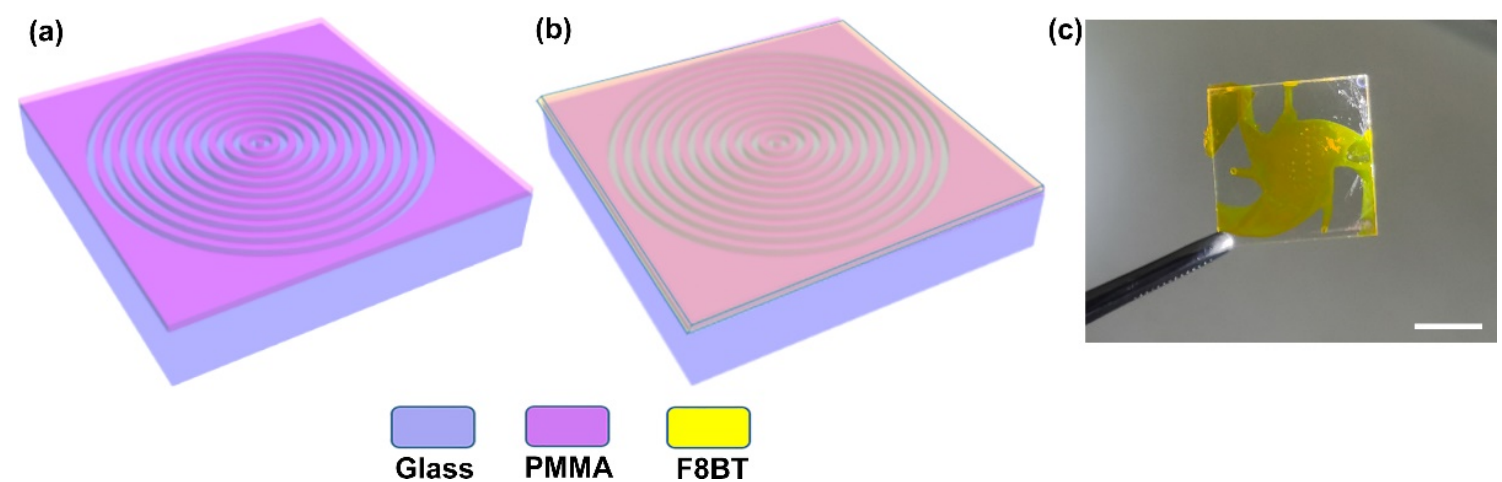

Figure 1. Schematic layout of the fabrication process. (a) The schematic layout of the circular periodic nanostructures written with the electron beam lithography (EBL). The blue-violet and purple color showing the glass substrate and electron beam resist PMMA film, respectively. (b) The schematic illustration of the gain material F8BT on the circular periodic geometry. The yellow color defines a gain medium F8BT film in the schematic layout. (c) The photograph of the actual composite; the scale bar is $1 \mathrm{~cm}$.

\section{Experiment Results}

Improved insight into the developed circular grating DFB polymer laser system can be gained by investigating its various properties such as morphology of the circular grating nanostructures, optical microscopic images, optical spectroscopic characterization, and lasing properties. The morphology of the circular grating structure was studied through the scanning electron microscope (SEM) to check the quality of the feedback cavity. The SEM photographs of the circular periodic nanostructures on the glass substrate at various magnification powers are depicted in the Figure 2a-d. As we can observe from the SEM photographs, the circular periodic nanostructures from high to low magnification power are free of defects and have considerable equal spacing between each modulation depth of the circular ring, indicating a significant interest in high-quality laser systems. In our experiment, the period of the circular grating was about $350 \mathrm{~nm}$ with respect to the second-order Brag equation used for DFB lasers.

Figure 3 displays dark and bright field optical micrograph of the circular periodic nanostructures at different magnification powers. The circular periodic geometry written with the EBL is highly uniform across the whole area of the gratings and shows high fidelity down to the sub-wavelength level, as can be observed from both the dark- and bright-field optical micrographs (Figure 3). 


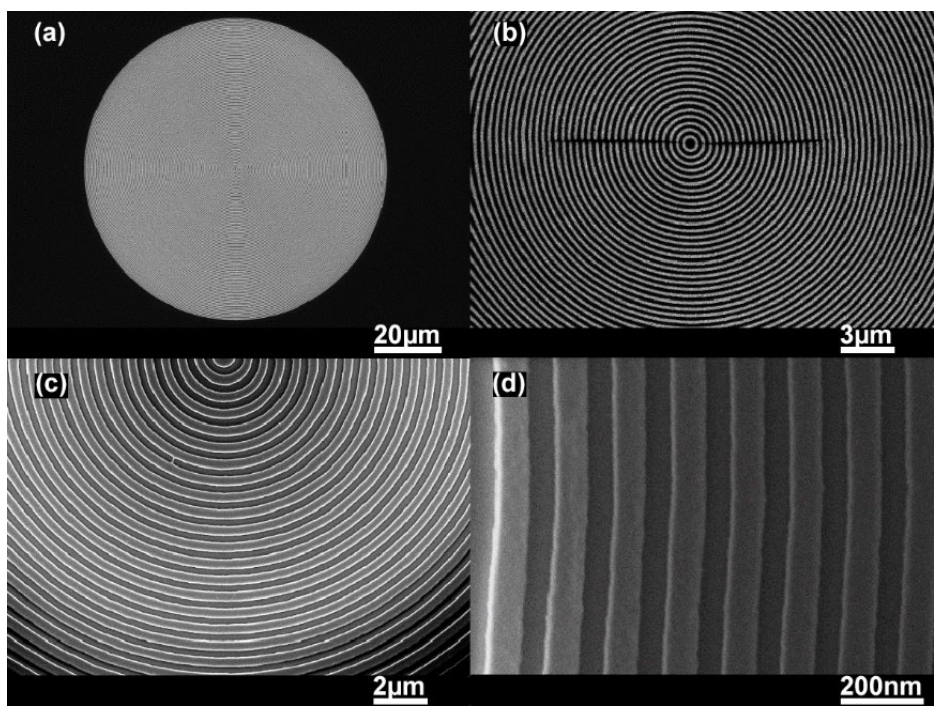

Figure 2. Scanning electron microscopic (SEM) images of the circular periodic nanostructures at (a) $1.03 \mathrm{k} \times$, (b) $6.74 \mathrm{k} \times$, (c) $12.29 \mathrm{k} \times$, and (d) $42.08 \mathrm{k} \times$ magnifications, respectively, showing the well-defined morphology of the respective ring cavities.

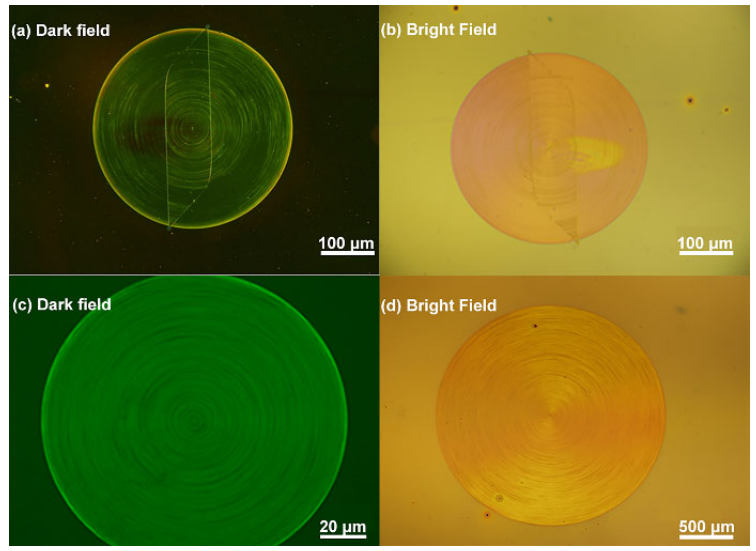

Figure 3. Optical microscopic images of the circular periodic structures. (a) and (c): Dark-field optical micrographs. (b) and (d): Bright-field optical micrographs.

In our experiment, we spin-coated a thin film of F8BT on the circular and regular (1D) grating structures to study the angle resolved polarization properties of the TE mode. Both the samples were excited with a non-polarized white light from tungsten halogen lamp (HL-2000, Ocean Optics, FL, USA). The optical transmission spectra were measured by using fiber spectrum analyzer from optical spectrometer (USB 4000, Ocean Optics, FL, USA). The incident white light can be TE polarized, when its electric field is parallel to the incident grating planes. Extinction spectrum for both the circular and regular gratings (one dimension, 1D) covered with a gain material F8BT can be achieved from the formula $-\log \left(I_{t} / I_{0}\right)$, where $I_{t}$ and $I_{o}$ indicate the intensities of the transmitted spectrum, as shown in the curves of Figure $4 a, b$. The wide peaks in both the Figure $4 a, b$ correspond to absorption spectrum of the conjugated polymer F8BT, whereas the narrow peaks show the coupling between the Bragg diffraction from the periodic structures (circular and regular) and waveguide (F8BT film) mode [23,39]. From the Figure $4 a$, it can be seen that by increasing the angle with a step of $45^{\circ}$ there is no change in the extinction curves. These phenomena are observed due to the symmetric distribution of the periodic structures and efficient coupling between the circular concentric rings and waveguide mode, while Figure $4 \mathrm{~b}$ represents the change in the extinction spectra by increasing the incident angle for the 1D regular grating geometry. A narrow peak of the extinction graph (in Figure $4 b$ ) would represent maximum intensity 
when the regular grating structure plane and incident white light electric field are parallel $\left(\theta=0^{\circ}\right.$ and $\left.180^{\circ}\right)$ to each other. Therefore, the circular periodic geometry combined with the significant optical properties of the conjugated polymer F8BT as a gain medium can improve the lasing performance of the DFB polymer lasers.
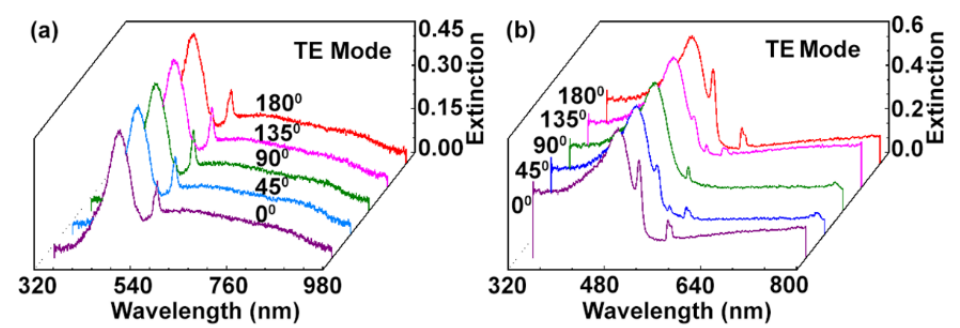

(c)

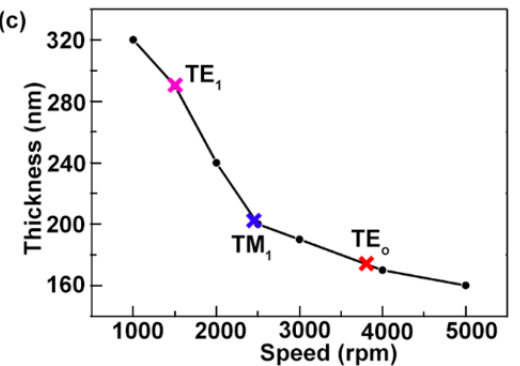

Figure 4. Optical characterization of the circular periodic grating and regular periodic grating. (a) Extension spectra of the circular grating at different polarization angle for $\mathrm{TE}_{0}$ mode. (b) Regular grating extinction curve indicating polarization at various angles for $\mathrm{TE}_{0}$ mode. (c) Thickness of the F8BT film as a function of spin-coating speed. The red, blue, and pink colors in the F8BT thickness curve represent $\mathrm{TE}_{0}, \mathrm{TM}_{1}$ and $\mathrm{TE}_{1}$ mode availability, according to the variation of the film thickness.

In our experiment, we studied the single as well as $\mathrm{TE}_{0}$ lasing mode emitted from the circular grating polymer laser. For this purpose, we spin-coated F8BT on a glass substrate at different spin-coating speeds. Therefore, the relationship between the various spin-coating speeds and the F8BT film thickness is depicted in the Figure 3c. The thickness of the waveguide plays an important role in the emission of lasing mode. Therefore, the necessary critical thickness $d_{e}$ (TE mode critical thickness) and $d_{m}$ (TM mode critical thickness) for the waveguide (F8BT film) can be calculated from the below equations [40]:

$$
\begin{gathered}
d_{e}=\frac{\left(2 p_{e}+1\right) \lambda}{4 \sqrt{n_{w g}^{2}-1}},\left(p_{e}=0,1,2 \ldots \ldots\right) \\
d_{m}=\frac{p_{m} \lambda}{2 \sqrt{n_{w g}^{2}-1}},\left(p_{m}=1,2,3 \ldots \ldots\right)
\end{gathered}
$$

In the above equations, $p_{e}$ and $p_{m}$ are the $p$ th order of TE and TM modes. The other parameters such as $\lambda$ and $n_{w g}$ are the emission wavelength and refractive index of the waveguide, respectively. Similarly, for a given experiment in which $n_{g}=1.8$ (refractive index of F8BT), $\lambda=577 \mathrm{~nm}$ (emission wavelength), and $p_{e}=0$, the critical thickness was about $96 \mathrm{~nm}$. Hence, above this thickness one $\mathrm{TE}_{0}$ mode exists in the F8BT waveguide. For $\mathrm{TM}_{1}$ and $\mathrm{TE}_{1}$, mode the critical thickness of the F8BT film would be greater than $192 \mathrm{~nm}$ and $289 \mathrm{~nm}$, respectively. The red, blue, and pink cross in the Figure $3 \mathrm{c}$ indicates the F8BT film critical thickness range for $\mathrm{TE}_{0}, \mathrm{TM}_{1}$, and $\mathrm{TE}_{1}$.

To underline the lasing emission properties, the measurements from the circular DFB polymer laser were carried out under optical pumping. Figure 5 a illustrates the schematic of the optical layout used for the optical pumping. In this experiment, the prepared sample of circular grating polymer laser was excited with a frequency-doubled Ti:sapphire laser (400 $\mathrm{nm}$ wavelength, $1 \mathrm{Khz}$ repetition rate, $200 \mathrm{fs}$ pulse duration). The optical pump beam 
after passing through the optical layout incident perpendicularly on the sample facing F8BT side toward the pump beam and the emission spectrum were recorded on the other side of the sample with fiber spectrometer (Maya 2000 Pro, Ocean Optics), as shown in Figure 5a. As we discussed at the beginning of the experiment, we designed our circular grating polymer laser according to the second-order DFB laser. Similarly, the single $\mathrm{TE}_{0}$ mode lasing was recorded at $577 \mathrm{~nm}$, as illustrated in Figure 5b. When the excitation energy of the pump source is low (below the threshold, black line in the Figure 5b), very weak emission is recorded similar to the photoluminescence (PL) spectra. We detected a surface-emitting single- and zero-order TE modes with the increase of pump excitation energy above the threshold (see Figure $5 b, c$ ). Therefore, a sharp linear growth of the output emission intensity provided more insight into the lasing characteristics, as depicted in the Figure 5c. Lasing action can be observed in the circular grating DFB polymer laser with a minimum threshold of $19 \mu \mathrm{J} / \mathrm{cm}^{2}$, which is the lowest threshold as compared to previously reported circular grating DFB lasers DFB lasers [34,37]. In Figure 5c, the blue and red spherical balls define the intensity of emission spectra below and above the threshold, whereas the black arrow is the threshold point and the linear fit is indicated by a blue and red (below and above threshold) line for the guidance of the readers (see Figure 5c).

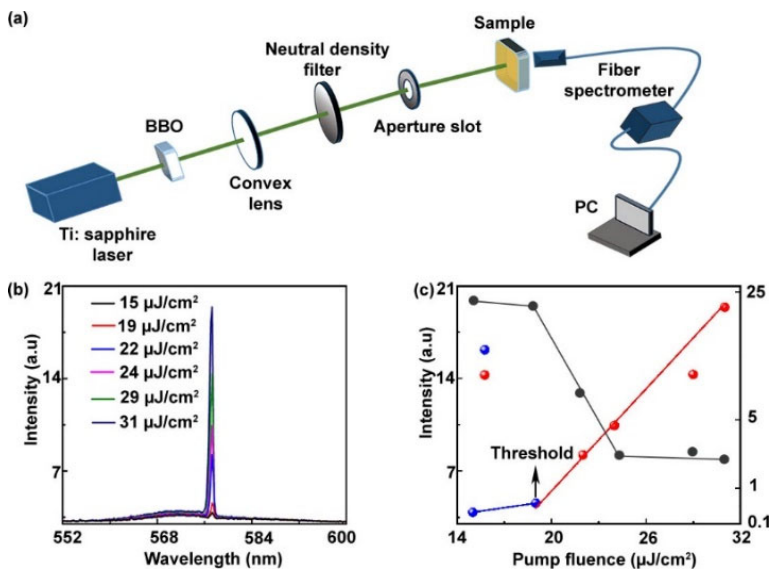

Figure 5. Lasing behavior of the circular periodic DFB polymer lasers. (a) Schematic optical layout out used for pumping lasing device. (b) Optical emission spectra at different pump fluences. (c) Evolution of the output intensity and the full width at half maxima as function of pump fluence. The black arrow in the graph indicates the threshold of the circular periodic DFB polymer laser about $19 \mu \mathrm{J} / \mathrm{cm}^{2}$

\section{Conclusions}

In summary, we have fabricated a single-mode circular grating DFB polymer laser by combining electron beam lithography and the spin-coating technique. The critical thickness for the zero-order TE mode was estimated with the formula given in the literature. Therefore, a convenient and easy fabrication (spin-coating) technique was applied to realize a very thin film of about $110 \mathrm{~nm}$ from the gain material F8BT. The optical extinction spectra section in our experiment can provide sufficient information about the efficient enhancement of the circular periodic geometry with the photoluminescence spectrum of the conjugated polymer F8BT. As a result, we have realized low-threshold (approximately $19 \mathrm{\mu J} / \mathrm{cm}^{2}$ ) single- and zero-order modes with a surface-emitting coherent beam in the circular grating DFB polymer laser. We expect that such a low threshold lasing device can find application in optoelectronic devices. 
Author Contributions: Conceptualization, S.C. and T.Z.; Methodology, S.C. and F.C.; Validation, S.C., T.Z.; Formal Analysis, A.H. and T.Z.; Investigation, F.C. and S.C.; Writing-Original Draft Preparation, A.H.; Writing-Review and Editing, A.H., S.C. and T.Z.; Supervision, T.Z.; Project Administration, T.Z.; Funding Acquisition, T.Z. All authors have read and agreed to the published version of the manuscript.

Funding: National Natural Science Foundation of China (NSFC) (61822501, 11504010); National Key R \& D Program of China (Grant No. 2017YFC0110203).

Institutional Review Board Statement: Not applicable.

Informed Consent Statement: Not applicable.

Data Availability Statement: Data sharing not available.

Acknowledgments: The authors would like to acknowledge National Natural Science Foundation of China (NSFC) (61822501, 11504010) and National Key R \& D Program of China (Grant No. 2017YFC0110203) for financial support.

Conflicts of Interest: The authors declare no conflict of interest.

\section{References}

1. Van der Ziel, J.; Tsang, W.; Logan, R.; Mikulyak, R.; Augustyniak, W. Subpicosecond pulses from passively mode-locked GaAs buried optical guide semiconductor lasers. Appl. Phys. Lett. 1981, 39, 525-527. [CrossRef]

2. San Miguel, M.; Feng, Q.; Moloney, J.V. Light-polarization dynamics in surface-emitting semiconductor lasers. Phys. Rev. A 1995, 52, 1728. [CrossRef]

3. Tessler, N.; Denton, G.; Friend, R. Lasing from conjugated-polymer microcavities. Nature 1996, 382, 695-697. [CrossRef]

4. Khairutdinov, R.; Serpone, N. Photophysics of cyanine dyes: Subnanosecond relaxation dynamics in monomers, dimers, and H-and J-aggregates in solution. J. Phys. Chem. B 1997, 101, 2602-2610. [CrossRef]

5. Zhai, T.; Zhou, Y.; Chen, S.; Wang, Z.; Shi, J.; Liu, D.; Zhang, X. Pulse-duration-dependent and temperature-tunable random lasing in a weakly scattering structure formed by speckles. Phys. Rev. A 2010, 82, 023824. [CrossRef]

6. Yap, B.K.; Xia, R.; Campoy-Quiles, M.; Stavrinou, P.N.; Bradley, D.D. Simultaneous optimization of charge-carrier mobility and optical gain in semiconducting polymer films. Nat. Mater. 2008, 7, 376-380. [CrossRef]

7. Kan, S.C.; Vassilovski, D.; Wu, T.C.; Lau, K.Y. Quantum capture limited modulation bandwidth of quantum well, wire, and dot lasers. Appl. Phys. Lett. 1993, 62, 2307-2309. [CrossRef]

8. Rong, K.; Sun, C.; Shi, K.; Gong, Q.; Chen, J. Room-temperature planar lasers based on water-dripping microplates of colloidal quantum dots. ACS Photon. 2017, 4, 1776-1784. [CrossRef]

9. Veldhuis, S.A.; Boix, P.P.; Yantara, N.; Li, M.; Sum, T.C.; Mathews, N.; Mhaisalkar, S.G. Perovskite materials for light-emitting diodes and lasers. Adv. Mater. 2016, 28, 6804-6834. [CrossRef]

10. Turnbull, G.; Andrew, P.; Barnes, W.L.; Samuel, I. Photonic mode dispersion of a two-dimensional distributed feedback polymer laser. Phys. Rev. B 2003, 67, 165107. [CrossRef]

11. Reufer, M.; Riechel, S.; Lupton, J.; Feldmann, J.; Lemmer, U.; Schneider, D.; Benstem, T.; Dobbertin, T.; Kowalsky, W.; Gombert, A. Low-threshold polymeric distributed feedback lasers with metallic contacts. Appl. Phys. Lett. 2004, 84, 3262-3264. [CrossRef]

12. Virgili, T.; Lidzey, D.; Grell, M.; Bradley, D.; Stagira, S.; Zavelani-Rossi, M.; De Silvestri, S. Influence of the orientation of liquid crystalline poly $(9,9$-dioctylfluorene) on its lasing properties in a planar microcavity. Appl. Phys. Lett. 2002, 80, 4088-4090. [CrossRef]

13. Giovanella, U.; Betti, P.; Bolognesi, A.; Destri, S.; Melucci, M.; Pasini, M.; Porzio, W.; Botta, C. Core-type polyfluorene-based copolymers for low-cost light-emitting technologies. Org. Electron. 2010, 11, 2012-2018. [CrossRef]

14. Kozlov, V.; Parthasarathy, G.; Burrows, P.E.; Khalfin, V.; Wang, J.; Chou, S.; Forrest, S. Structures for organic diode lasers and optical properties of organic semiconductors under intense optical and electrical excitations. IEEE J. Quantum Electron. 2000, 36, 18-26. [CrossRef]

15. Scherf, U.; Riechel, S.; Lemmer, U.; Mahrt, R. Conjugated polymers: Lasing and stimulated emission. Curr. Opin. Solid State Mater. Sci. 2001, 5, 143-154. [CrossRef]

16. Kawabe, Y.; Spiegelberg, C.; Schülzgen, A.; Nabor, M.; Kippelen, B.; Mash, E.; Allemand, P.; Kuwata-Gonokami, M.; Takeda, K.; Peyghambarian, N. Whispering-gallery-mode microring laser using a conjugated polymer. Appl. Phys. Lett. 1998, 72, 141-143. [CrossRef]

17. Kushida, S.; Okada, D.; Sasaki, F.; Lin, Z.H.; Huang, J.S.; Yamamoto, Y. Low-threshold whispering gallery mode lasing from self-assembled microspheres of single-sort conjugated polymers. Adv. Opt. Mater. 2017, 5, 1700123. [CrossRef]

18. Sun, S.; Murray, C. Synthesis of monodisperse cobalt nanocrystals and their assembly into magnetic superlattices. J. Appl. Phys. 1999, 85, 4325-4330. [CrossRef]

19. Zhai, T.; Cao, F.; Chu, S.; Gong, Q.; Zhang, X. Continuously tunable distributed feedback polymer laser. Opt. Express 2018, 26, 4491-4497. [CrossRef] 
20. Cao, F.; Niu, L.; Tong, J.; Li, S.; Hayat, A.; Wang, M.; Zhai, T.; Zhang, X. Hybrid lasing in a plasmonic cavity. Opt. Express 2018, 26, 13383-13389. [CrossRef]

21. Tsutsumi, N.; Kawahira, T.; Sakai, W. Amplified spontaneous emission and distributed feedback lasing from a conjugated compound in various polymer matrices. Appl. Phys. Lett. 2003, 83, 2533-2535. [CrossRef]

22. Karl, M.; Glackin, J.M.; Schubert, M.; Kronenberg, N.M.; Turnbull, G.A.; Samuel, I.D.; Gather, M.C. Flexible and ultra-lightweight polymer membrane lasers. Nat. Commun. 2018, 9, 1525. [CrossRef]

23. Hayat, A.; Tong, J.; Chen, C.; Niu, L.; Aziz, G.; Zhai, T.; Zhang, X. Multi-wavelength colloidal quantum dot lasers in distributed feedback cavities. Sci. China Inf. Sci. 2020, 63, 182401. [CrossRef]

24. Samuel, I.D.; Turnbull, G.A. Polymer lasers: Recent advances. Mater. Today 2004, 7, 28-35. [CrossRef]

25. Chénais, S.; Forget, S. Recent advances in solid-state organic lasers. Polym. Int. 2012, 61, 390-406. [CrossRef]

26. Kuehne, A.J.; Gather, M.C. Organic lasers: Recent developments on materials, device geometries, and fabrication techniques. Chem. Rev. 2016, 116, 12823-12864. [CrossRef]

27. Samuel, I.D.W.; Turnbull, G.A. Organic semiconductor lasers. Chem. Rev. 2007, 107, 1272-1295. [CrossRef]

28. Fu, Y.; Zhai, T. Distributed feedback organic lasing in photonic crystals. Front. Optoelectron. 2020, 13, 18-34. [CrossRef]

29. Zhou, P.; Niu, L.; Hayat, A.; Cao, F.; Zhai, T.; Zhang, X. Operating characteristics of high-order distributed feedback polymer lasers. Polymers 2019, 11, 258. [CrossRef]

30. Erdogan, T.; King, O.; Wicks, G.; Hall, D.; Anderson, E.H.; Rooks, M. Circularly symmetric operation of a concentric-circle-grating, surface-emitting, AlGaAs/GaAs quantum-well semiconductor laser. Appl. Phys. Lett. 1992, 60, 1921-1923. [CrossRef]

31. Bauer, C.; Giessen, H.; Schnabel, B.; Kley, E.B.; Schmitt, C.; Scherf, U.; Mahrt, R. A Surface-Emitting Circular Grating Polymer Laser. Adv. Mater. 2001, 13, 1161-1164. [CrossRef]

32. Chen, Y.; Li, Z.; Zhang, Z.; Psaltis, D.; Scherer, A. Nanoimprinted circular grating distributed feedback dye laser. Appl. Phys. Lett. 2007, 91, 051109. [CrossRef]

33. Jordan, R.H.; Hall, D.G.; King, O.; Wicks, G.; Rishton, S. Lasing behavior of circular grating surface-emitting semiconductor lasers. J. Opt. Soc. Am. B 1997, 14, 449-453. [CrossRef]

34. Gao, Y.; Tobing, L.Y.; Kiffer, A.l.; Zhang, D.H.; Dang, C.; Demir, H.V. Azimuthally polarized, circular colloidal quantum dot laser beam enabled by a concentric grating. ACS Photon. 2016, 3, 2255-2261. [CrossRef]

35. Salerno, M.; Gigli, G.; Zavelani-Rossi, M.; Perissinotto, S.; Lanzani, G. Effects of morphology and optical contrast in organic distributed feedback lasers. Appl. Phys. Lett. 2007, 90, 111110. [CrossRef]

36. Erdogan, T.; Hall, D. Circularly symmetric distributed feedback semiconductor laser: An analysis. J. Appl. Phys. 1990, 68, 1435-1444. [CrossRef]

37. Prins, F.; Kim, D.K.; Cui, J.; De Leo, E.; Spiegel, L.L.; McPeak, K.M.; Norris, D.J. Direct patterning of colloidal quantum-dot thin films for enhanced and spectrally selective out-coupling of emission. Nano Lett. 2017, 17, 1319-1325. [CrossRef]

38. Zhai, T.; Tong, F.; Wang, Y.; Wu, X.; Li, S.; Wang, M.; Zhang, X. Polymer lasers assembled by suspending membranes on a distributed feedback grating. Opt. Express 2016, 24, 22028-22033. [CrossRef]

39. Zhai, T.; Wang, Y.; Liu, H.; Zhang, X. Large-scale fabrication of flexible metallic nanostructure pairs using interference ablation. Opt. Express 2015, 23, 1863-1870. [CrossRef]

40. Gianordoli, S.; Hainberger, R.; Köck, A.; Finger, N.; Gornik, E.; Hanke, C.; Korte, L. Optimization of the emission characteristics of light emitting diodes by surface plasmons and surface waveguide modes. Appl. Phys. Lett. 2000, 77, 2295-2297. [CrossRef] 